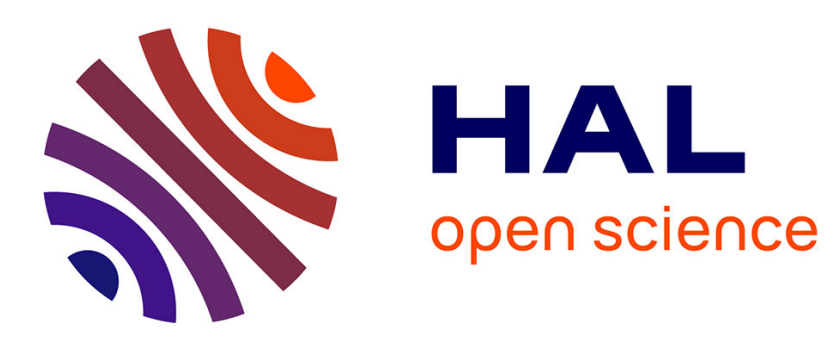

\title{
Experimental measurement of the Melnikov function
}

Patrice Meunier, Peter Huck, Clément Nobili, Emmanuel Villermaux

\section{To cite this version:}

Patrice Meunier, Peter Huck, Clément Nobili, Emmanuel Villermaux. Experimental measurement of the Melnikov function. Physics of Fluids, 2015, 27 (7), pp.077103. 10.1063/1.4927036 . hal-01219227

\section{HAL Id: hal-01219227 \\ https://hal.science/hal-01219227}

Submitted on 22 Oct 2015

HAL is a multi-disciplinary open access archive for the deposit and dissemination of scientific research documents, whether they are published or not. The documents may come from teaching and research institutions in France or abroad, or from public or private research centers.
L'archive ouverte pluridisciplinaire HAL, est destinée au dépôt et à la diffusion de documents scientifiques de niveau recherche, publiés ou non, émanant des établissements d'enseignement et de recherche français ou étrangers, des laboratoires publics ou privés. 


\title{
Experimental measurement of the Melnikov function
}

\author{
Patrice Meunier ${ }^{1}$, Peter Huck ${ }^{1}$, Clément Nobili ${ }^{1}$ \& Emmanuel Villermaux ${ }^{1,2}$ \\ 1) Aix Marseille Université, CNRS, Centrale Marseille, IRPHE UMR 7342, F-13384, \\ Marseille, France \\ ${ }^{2)}$ Institut Universitaire de France
}

(Dated: 8 July 2015)

We study the transport properties of a genuine two-dimensional flow with a large mean velocity perturbed periodically in time by means of an original experimental technique. The flow, generated by the co-rotation of two cylinders is both stratified with a linear density gradient using salted water, and viscous in order to prevent Ekman pumping and centrifugal instabilities. Thus, the mean flow contains a hyperbolic point with a homoclinic streamline, which we perturb periodically by an extra oscillation. A blob of scalar injected close to the stagnation point contracts on the stable manifold, and stretches in the unstable direction. The distance between the stable and the unstable manifolds is measured as the distance between the maximum and the minimum of the dye undulating pattern, and is recorded as a function of the perturbation frequency. This distance, also called the Melnikov function, presents a maximum when the residence time of a fluid particle in the mean flow is about half a perturbation period. This resonance criterion is recovered with good quantitative agreement by the theoretical prediction of the Melnikov function computed for this flow.

\section{INTRODUCTION}

Scalar mixing is a key point for numerous natural and man-made operations, spanning a broad range of length scales from microns ${ }^{1,2}$ to geophysical scales ${ }^{3}$. In the atmosphere, fluxes of moisture, temperature and chemical components are governed by mixing. Scalars can be trapped in atmospheric vortices, thus representing barriers for transport ${ }^{4-7}$. For example, strong polar vortices form above each pole during winters, and maintain low temperatures and low ozone concentrations. However, it was observed that these polar vortices may split, as happened in 2002 on the Antarctic polar vortex ${ }^{8}$, creating undulating stretched structures leading to fast mixing ${ }^{9}$. The dynamics in this instance was governed by the presence of a hyperbolic point with a homoclinic manifold i.e. whose streamline reconnects on itself. This object, which consists of a weak unsteady flow added to a hyperbolic point flow, has been long ago identified as a paradigm in chaotic advection ${ }^{10-12}$, but despite its fame has never been the subject of laboratory study. The goal of the present study is to reproduce experimentally this type of flow and to analyse it with available theories, namely Melnikov theory.

The dynamics homoclinic and heteroclinic manifolds has been widely studied in dynamical systems theory ${ }^{13,14}$. Indeed, the stability of these trajectories with respect to external perturbations is of critical importance in the transition to chaotic behaviors. This problem is often studied as an hamiltonian time-invariant system perturbed by a small time-periodic flow. The homoclinic (or heteroclinic) trajectory splits into a stable and an unstable manifold whose separation distance can be predicted at first order by a simple integral called the Melnikov function ${ }^{13}$. This theory was then applied in fluid dynamics ${ }^{11}$ in the case of two counter-rotating point vortices in order to predict the transport of scalar across the homoclinic trajectory. It is a very interesting result since this slow transport across the separatrix is usually the limiting process of the long-range transport in chaotic mixing ${ }^{15}$. This powerful technique was used for numerical and theoretical studies of various flows ${ }^{16-21}$. Such a mechanism was proven to be responsible for large asymmetries in the dye visualisations of vortex breakdown ${ }^{22,23}$ and lead to strong transport from inside to outside the vortex breakdown bubble ${ }^{24,25}$. However, this technique has never been used to quantitatively predict the transport of a real experimental flow except once ${ }^{25}$, where it was found to be an order of magnitude too large. 


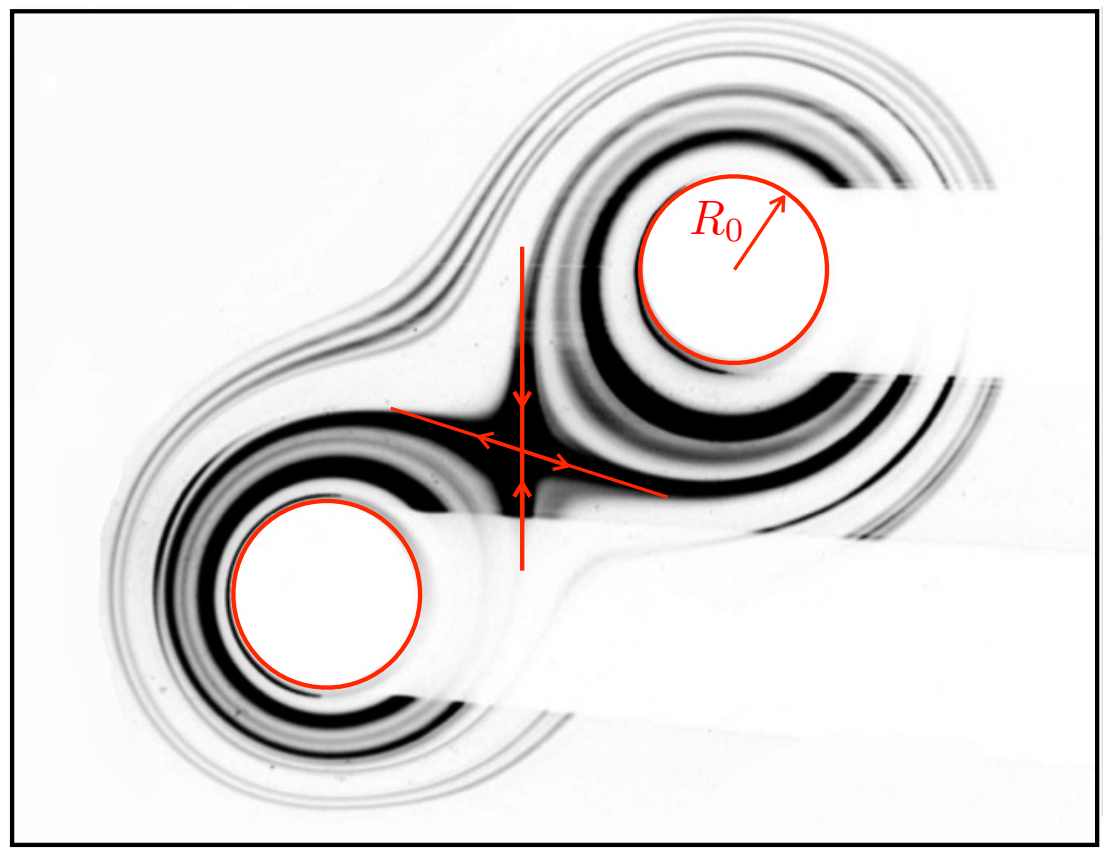

FIG. 1: Experimental set-up showing the streamlines around two cylinders of radii $R_{0}$, co-rotating anti-clockwise. The flow features a tilted saddle point at the mid-distance between the cylinders centers, separated by $D$. A plane laser sheet illuminates an isopycnal, from left to right. The dye is fluorescein, density matched at the plane of illumination. The perturbing oscillating cylinder with radius $R_{1}$ is shown in Fig. 2 .

Early experimental studies have focused on two-dimensional (2D) flows which would oscillate temporally between two states ${ }^{26-30}$, and showed that the Poincaré sections ${ }^{31}$ could distinguish the zones of high (chaotic) dispersion from the zones where the particles were trapped (KAM tori and periodic orbits). Solomon and Gollub (1988) ${ }^{32}$ also showed that a periodic oscillation of the flow would enhance the advection of a passive scalar in RayleighBenard convection rolls. But all these flows were obtained in a very viscous fluid where Ekman pumping on the boundaries might have introduced an additional three-dimensional (3D) motion.

In order to keep the flow two-dimensional, experimentalists have tried to confine the flow in a thin layer of electrolyte forced electromagnetically ${ }^{33-38}$. But the flow would still be subject to a 3D Ekman pumping at large amplitudes ${ }^{39}$ (although reduced thanks to the stratification of the electrolyte ${ }^{40}$ ), leading to a complete and quicker mixing ${ }^{41}$. The idea of using a stratified flow has been used in the case of a vortex ${ }^{42}$ which increased by a factor ten the lifetime of the vortex. However, stratified flows are also subject to 3D instabilities at large Reynolds numbers. This paper presents a new experimental technique which allows the motions to remain exactly $2 \mathrm{D}$ over a very long time.

The paper is organized as follows: after presenting the experimental set-up in section II, the flow is analyzed in detail in section III before the Melnikov function is measured in section IV. Conclusions follow in section V. 


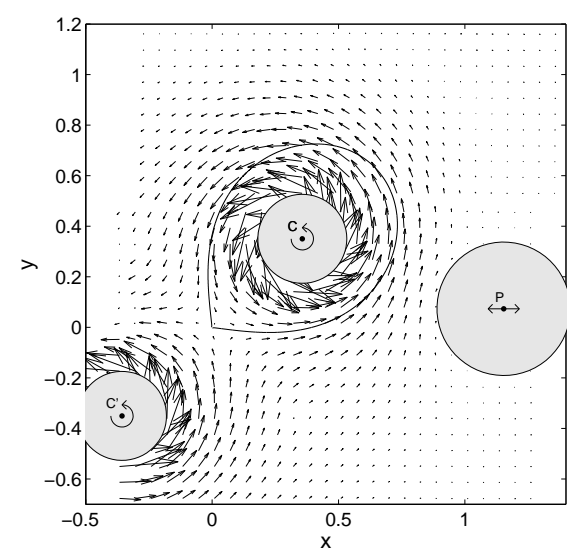

(a)

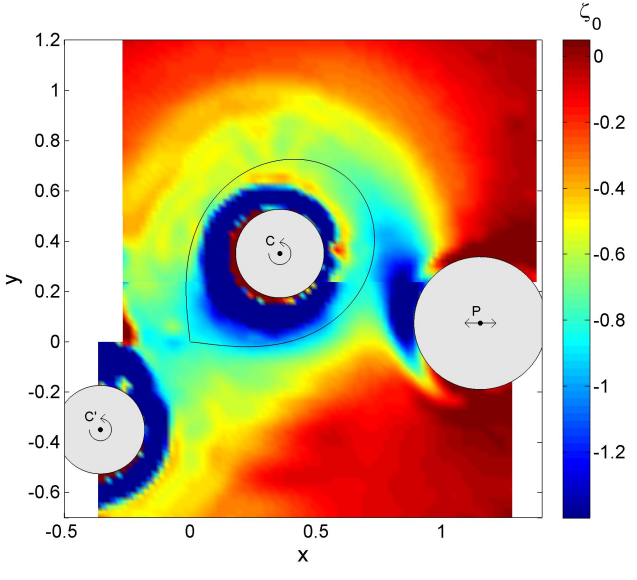

(b)

FIG. 2: Mean velocity (a) and (b) vorticity field for $\omega=2.47, \epsilon=0.35$ and $R e=125$. The rotating cylinders are centered in $C$ and $C^{\prime}$ and the oscillating cylinder is centered in $P$.

\section{FORMULATION OF THE PROBLEM}

\section{A. Periodic perturbation of a homoclinic point}

The goal is to study the Melnikov theory in a real experiment by creating a flow with a homoclinic point under the influence of a periodic perturbation. This is done by rotating at the angular velocity $\omega_{0}=0.5-2 \mathrm{rad} / \mathrm{s}$ two cylinders of radius $R_{0}=2 \mathrm{~cm}$ separated by a distance $D=11.3 \mathrm{~cm}$ between their axes (see Fig. 1). This creates a hyperbolic point at the middle between the cylinders whose streamlines surround each cylinder. This mean 2D incompressible (i.e. Hamiltonian viewed from dynamical systems) flow is perturbed periodically by a third cylinder of radius $R_{1}=3 \mathrm{~cm}$ which oscillates in translation at the frequency $\omega_{1}=0.3-2 \mathrm{rad} / \mathrm{s}$ with an amplitude $A$. This flow is contained in a Plexiglas tank of dimensions $40 \times 40 \times 16 \mathrm{~cm}^{3}$ to allow visualisations from the side and from the top.

All lengths are counted in units of the distance $D$ between the rotating cylinders. This defines a first non-dimensional parameter $r_{0}=R_{0} / D=0.177$, which is small. As a consequence, the flow close to each rotating cylinder is similar to a point vortex flow (decaying as $1 / r)$ with a circulation $\Gamma=2 \pi R_{0}^{2} \omega_{0}$. This circulation $\Gamma$ is used to define the timescale $D^{2} / \Gamma$. The two main non-dimensional parameters are thus the non-dimensional frequency and amplitude of the forcing

$$
\omega=\frac{\omega_{1}}{\Gamma / D^{2}} \quad \text { and } \quad \epsilon=\frac{A}{D}
$$

The flow also depends on the ratio $r_{1}=R_{1} / D=0.265$ which is kept constant in this study. The Reynolds number $R e=\Gamma / \nu=125-500$ ( $\nu$ being the fluid kinematic viscosity) has no impact on the flow.

\section{B. 2D or not $2 \mathrm{D}$ ?}

That is the question of any experimentalist trying to study a 2D flow in our 3D world. Several attempts have been made by studying a thin electrolyte layer electromagnetically forced or by using a soap film. In the first case, the friction from the bottom damps the flow and eventually enables vertical Ekman pumping at large Reynolds number. In the second 


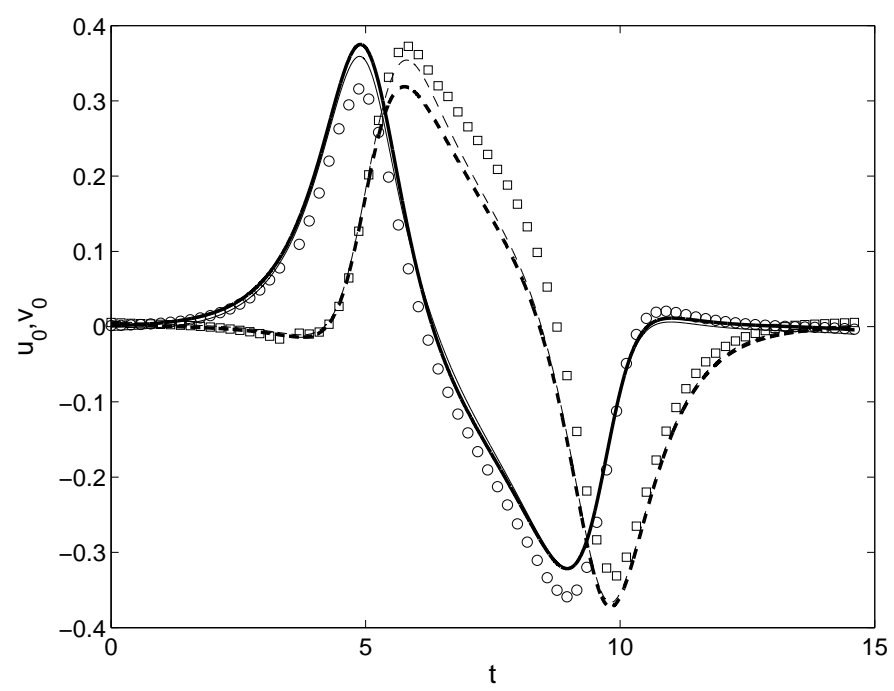

FIG. 3: Profile of velocity component $u_{0}=\partial \Psi_{0} / \partial y$ (solid line, o) and velocity component $v_{0}=-\partial \Psi_{0} / \partial x$ (dashed line, $\square$ ) along the homoclinic streamline. Symbols $(\circ, \square)$

correspond to experimental data obtained by PIV measurements whereas solid and dashed lines correspond the theoretical prediction of Eq. (2) without (thick lines) and with (thin lines) the correction given by Eq. (3) induced by the oscillating cylinder. Here, $\omega=2.47$,

$$
\epsilon=0.35 \text { and } R e=125 \text {. }
$$

case, the variation of the thickness of the soap film creates some divergence in the flow as in a compressible 2D flow. Both set-ups are thus not ideal to create a $2 \mathrm{D}$ incompressible flow.

In a previous study ${ }^{42}$, we had used a stable density gradient to prevent any vertical velocity. Indeed, imposing a continuous linear variation of the density with the altitude allowed to study the mixing properties of a solitary vortex which remained $2 \mathrm{D}$ during a few hundred revolutions. We used the same set-up in this study, but we increased the viscosity of the fluid to prevent a 3D centrifugal instability to appear. For this purpose, we added to the water $10 \%$ by volume of $\mathrm{UCON}^{\mathrm{TM}}$ oil $^{43}$ whose viscosity $\nu_{\mathrm{Ucon}}$ is about $10^{4}$ that of water at $20^{\circ}$ Celsius, in order to increase the fluid viscosity by a factor about 10 . This allowed to decrease the Reynolds number below the threshold of the centrifugal instability while allowing to stratify the water by adding salt.

The continuous stratification is obtained using the double bucket method. The solution of water and $\mathrm{UCON}^{\mathrm{TM}}$ oil is split in two small buckets and $5 \%$ salt is added in one bucket. The salt-free solution is slowly injected at the bottom (under a $10 \mathrm{~cm}$ diameter disk) while the salted solution is continuously added to the salt-free solution (through a pipe connecting the two buckets). If the two buckets have the same area, the final fluid is stratified with a density varying linearly from the salted solution (at the bottom) to the salt-free solution (at the top). This stable density gradient could be used during two weeks for about a hundred experiments. The density gradient introduces an additional non-dimensional parameter, defined by the Froude number $F=\omega_{0} / N \sim 0.25-1$, where $N=\sqrt{-(g / \rho)(\partial \rho / \partial z)}$ is the buoyancy (Brunt-Väisälä) frequency. However, this parameter has no influence on the final results.

\section{Measurement techniques}

We used two different methods to study the velocity and the stirring properties of this flow. First, small reflecting particles were added into the fluid and illuminated by a hor- 
izontal laser sheet from a continuous Argon laser. Correlating two images separated by 0.3 seconds using a homemade Particle Image Velocimetry (PIV) algorithm ${ }^{44}$ permitted to get instantaneous velocity fields. Second, a small drop of dye (Fluoresceine) was injected at the altitude of the laser sheet close to the hyperbolic point. The dye stretches into a thin filament as it reaches the hyperbolic point. The filament is then advected around the rotating cylinder and thus reveals the unstable manifold of the homoclinic point. The characteristics of the unstable manifold were independent of the position of injection of the dye. Because of the Stokes-Einstein law, the diffusivity of Fluorescein in a UCON ${ }^{\mathrm{TM}}$ oil/water mixture 10 times more viscous than pure water is 10 times smaller than the diffusivity of Fluorescein in water, i.e. of the order of $\kappa=5 \times 10^{-11} \mathrm{~m}^{2} / \mathrm{s}$. This value was checked by measuring the traverse thickness $\sqrt{\kappa / \gamma}$ (Batchelor scale) of the filament in the stretching flow of the hyperbolic point of strength $\gamma \sim \Gamma / D^{2}$ from the temporal decrease of its maximum concentration $e^{-\gamma t}$. The corresponding Péclet number $P e=\omega_{0} R_{0}^{2} / \kappa=25-100 \times 10^{6}$ is very large. However, we do not focus in this study on the mixing properties of the flow (for which diffusion matters) but only on its stirring (advecting) properties. Our present discussion is thus independent of the Péclet number.

\section{BASE FLOW}

\section{A. Mean flow}

PIV measurements allowed to extract the instantaneous horizontal velocity fields which were then averaged in time. The mean velocity field is shown in Fig. 2(a) and clearly reveals the presence of the hyperbolic point at the middle between the rotating cylinders. The velocity is very strong and nearly axisymmetric around each rotating cylinder, which can be modeled by two point vortices with a circulation ${ }^{18} \Gamma=2 \pi R_{0}^{2} \omega_{0}$. However, a pair of two stationary point vortices is not a solution of the vorticity equation because a co-rotating vortex pair is rotating at the angular velocity $\Omega=\Gamma /\left(\pi D^{2}\right)$. The flow is thus modeled by a co-rotating vortex pair in the frame of reference rotating at $\Omega$, which imposes that the velocity is in solid body rotation at $-\Omega$ far from the cylinders. The stream function can be written in non-dimensional form

$$
\Psi_{0}=\frac{1}{4 \pi}\left(\log \left|\mathbf{r}-\mathbf{r}_{\mathbf{C}}\right|+\log \left|\mathbf{r}-\mathbf{r}_{\mathbf{C}^{\prime}}\right|-2|\mathbf{r}|^{2}\right)
$$

where the center $O$ of the reference frame is taken at the middle between the cylinders and where $\mathbf{r}_{\mathbf{C}}=(1,1) / \sqrt{2}$ and $\mathbf{r}_{\mathbf{C}^{\prime}}=(-1,-1) / \sqrt{2}$ correspond to the centers of the cylinders. The theoretical streamline starting at the hyperbolic point $O$ is plotted in Fig. 2(a) and shows a very good agreement with the experimental velocity field. By contrast with an earlier study ${ }^{18}$, the presence of the background rotation prevents an analytical derivation of the streamlines, which are thus calculated numerically.

The mean vorticity field $\zeta$ is plotted in Fig. 2(b). There is some negative vorticity close to the rotating cylinders of the order of unity. This is consistent with the mean theoretical vorticity which is uniform and equal to $-2 / \pi$. However, the vorticity decays far from the cylinders. This is probably due to the viscous friction at the bottom and to the presence of the oscillating cylinder which clearly modifies the mean velocity field (see Fig. 2a). However, along the streamline starting from the hyperbolic point $O$, the vorticity is close to the theoretical value within $20 \%$.

In order to quantitatively compare the theoretical and the experimental velocity field, we have plotted the mean velocity components $u_{0}=\partial \Psi_{0} / \partial y$ and $v_{0}=-\partial \Psi_{0} / \partial x$ along the streamline in Fig. 3. They are plotted as a function of time, i.e. for a fluid particle starting close to $O$. There is a fairly good agreement, although $v_{0}$ is slightly underestimated close to the oscillating cylinder. This is due to the deflection of the flow by the presence of the oscillating cylinder which accelerates the fluid. Indeed, the wake of the oscillating cylinder in the mean flow $\mathbf{u}_{0}$ creates a small correction which can be written (from the potential 


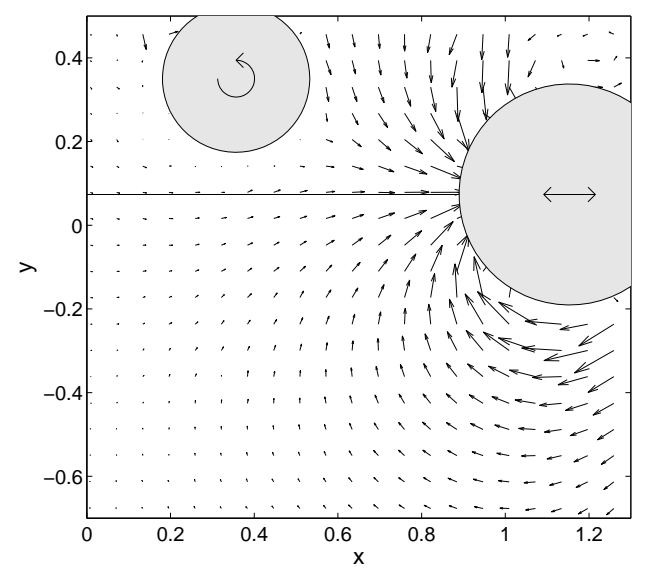

(a)

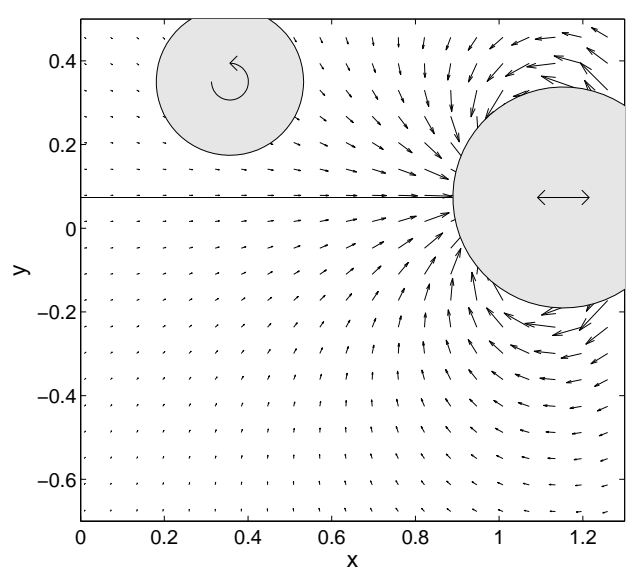

(b)

FIG. 4: Perturbation velocity field $\mathbf{u}_{\mathbf{1}}$ obtained (a) experimentally by phase-average and (b) theoretically from Eq. (4). $\omega=2.47, \epsilon=0.35$ and $R e=125$.

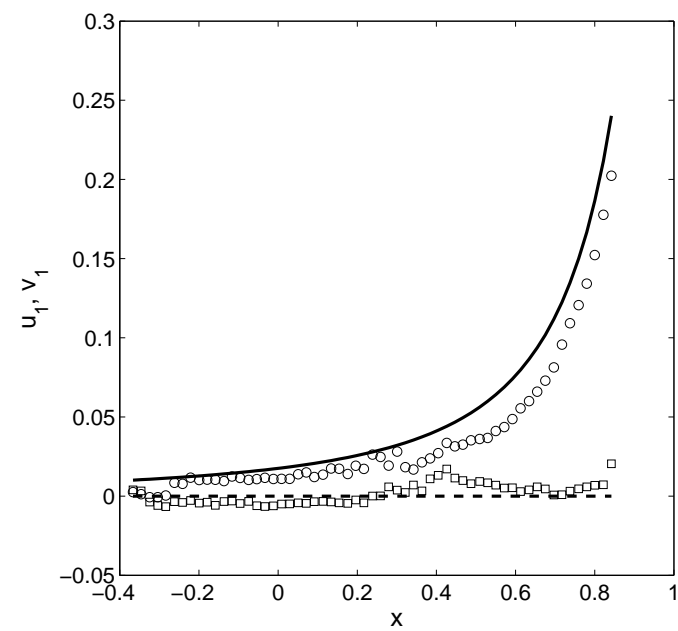

(a)

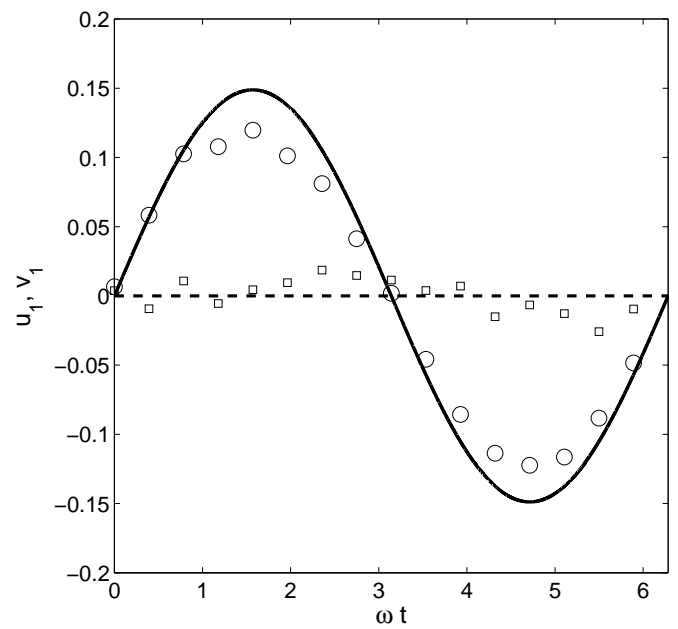

(b)

FIG. 5: Perturbation $u$ velocity (solid line, o) and $v$ velocity (dashed line, $\square$ ) obtained experimentally (lines) and theoretically (symbols). (a) $x$-profile at $y=0.07$ (indicated by a solid line on Fig. 4. (b) Temporal variation. $\omega=2.47, \epsilon=0.35$ and $R e=125$.

flow around a cylinder ${ }^{45}$ )

$$
\mathbf{u}_{\text {corr }}=\left|\mathbf{u}_{0}\left(\mathbf{r}_{\mathbf{p}}\right)\right| \frac{r_{1}^{2}}{\left|\mathbf{r}-\mathbf{r}_{p}\right|^{2}}\left(\begin{array}{c}
\cos \left(2 \theta_{\text {corr }}\right) \\
\sin \left(2 \theta_{\text {corr }}\right)
\end{array}\right) \text {. }
$$

Here $\theta_{\text {corr }}$ is the angle between the vector $\mathbf{r}-\mathbf{r}_{p}$ and the mean velocity $\mathbf{u}_{\mathbf{0}}\left(\mathbf{r}_{\mathbf{p}}\right)$ at the center $\mathbf{r}_{p}=\left(x_{p}, y_{p}\right)=(1.15,0.07)$ of the oscillating cylinder. Adding this mean correction to the mean flow creates a velocity along the streamline which is plotted as thin lines in Fig. 3, in better agreement with the experimental data. 


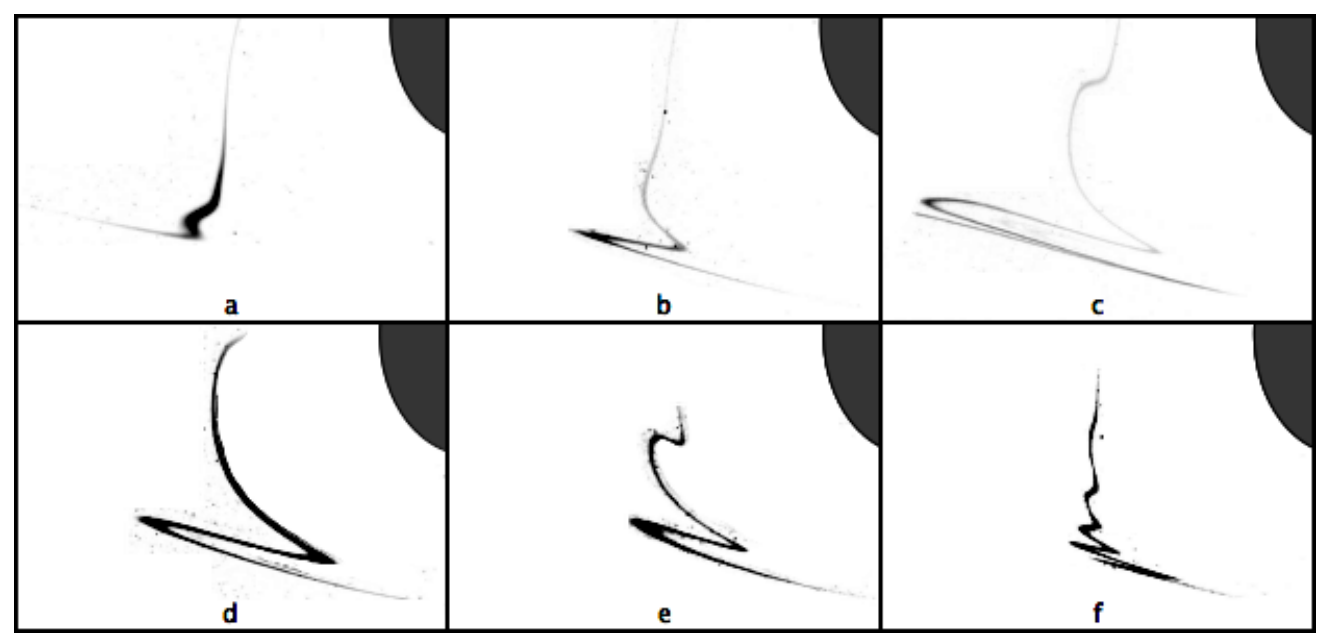

FIG. 6: Visualisation of the dye injected close to the hyperbolic point after one rotation around the rotating cylinder visible in the top right corner of the image. Top row: $\omega_{1}=1.36 \mathrm{rad} / \mathrm{s}$ and $\omega_{0}=2.00 \mathrm{rad} / \mathrm{s}$ are held constant while the perturbation amplitude A is varied. (a) $A=0.006$, (b) $A=0.011$, (c) $A=0.024$. Bottom row: Rotating frequency $\omega_{0}=1.00 \mathrm{rad} / \mathrm{s}$ and perturbation amplitude $A=0.024$ are held constant while the perturbation frequency is varied. (d) $\omega_{1}=0.63 \mathrm{rad} / \mathrm{s}(\mathrm{e}) \omega_{1}=1.15 \mathrm{rad} / \mathrm{s}$ (f) $\omega_{1}=1.99$ $\mathrm{rad} / \mathrm{s}$.

\section{B. Periodic perturbation}

The flow created by the oscillation of the cylinder can be measured experimentally by phase averaging the instantaneous velocity fields and then subtracting the mean flow. It is plotted in Fig. 4(a) at a phase where the cylinder is moving toward positive $x$.

This flow is again modeled as the potential flow around a cylinder (as for the correction of the mean flow). In non-dimensional form, the cylinder has a radius $r_{1}$ and is oscillating in the $x$ direction at the frequency $\omega$ with an amplitude $\epsilon$. The oscillating flow can thus be written in cartesian coordinates (here $\theta_{p}$ is the angle between the vector $\mathbf{r}-\mathbf{r}_{p}$ and the $x$-axis)

$$
\mathbf{u}_{1}=\epsilon \omega \cos (\omega t) \frac{r_{1}^{2}}{\left|\mathbf{r}-\mathbf{r}_{p}\right|^{2}}\left(\begin{array}{c}
\cos \left(2 \theta_{p}\right) \\
\sin \left(2 \theta_{p}\right)
\end{array}\right) .
$$

This velocity field is plotted in Fig. 4(b) and is in excellent qualitative agreement with the experimental velocity field. There is only a minor discrepancy above the cylinder. This is probably due to the shade created by the cylinder in the laser sheet, which alters the PIV measurements.

A quantitative comparison between these two fields can be done by taking velocity profiles along the solid line (passing through the center of the oscillating cylinder). It is plotted in Fig. 5(a) and shows that the theory overestimates the $v_{1}$ velocity by about $20 \%$. However, this may come from the noise in the measurements which is large because $\mathbf{u}_{\mathbf{1}}$ is a first order velocity much smaller than $\mathbf{u}_{\mathbf{0}}$. The temporal variation of this velocity is plotted in Fig. 5(b) which confirms that the velocity is oscillating sinusoidally in time. There is again a small discrepancy between the experiment and the theory which slightly overestimates $v_{1}$. 


\section{MELNIKOV FUNCTION}

In order to quantify the transport properties of this flow, a blob of dye is injected close to the hyperbolic point $O$. The dye stretches along the unstable manifold, is advected around the rotating cylinder and comes back to the hyperbolic point $O$. Fig. 6 shows the shape of the dye filament when it reaches the hyperbolic point for various amplitudes $\epsilon$ and oscillating frequencies $\omega$. It exhibits undulations whose amplitude is larger for larger oscillations of the cylinder. Moreover, the wavelength is smaller when the oscillating frequency is larger.

As sketched in Fig. 7, these undulations are due to the perturbing flow $\mathbf{u}_{1}$ of the oscillating cylinder. Intuitively, the fluid particles are advected between point $\mathrm{D}$ and $\mathrm{E}$ to the right or to the left depending on the phase of the perturbation. The fluid particle is thus displaced a distance $\delta$ at point $E$. This distance to the unperturbed streamline increases when the fluid particle reaches the hyperbolic point $O$. This is clearly visible in Fig. 8(a) where the undulations grow in amplitude close to the hyperbolic point. The maxima and minima of these undulations have been measured on several images. They are plotted in Fig. 8(b) and seem to be located on a hyperbola although based on non-orthogonal axes $\left(x^{\prime}, y^{\prime}\right)$. Indeed,

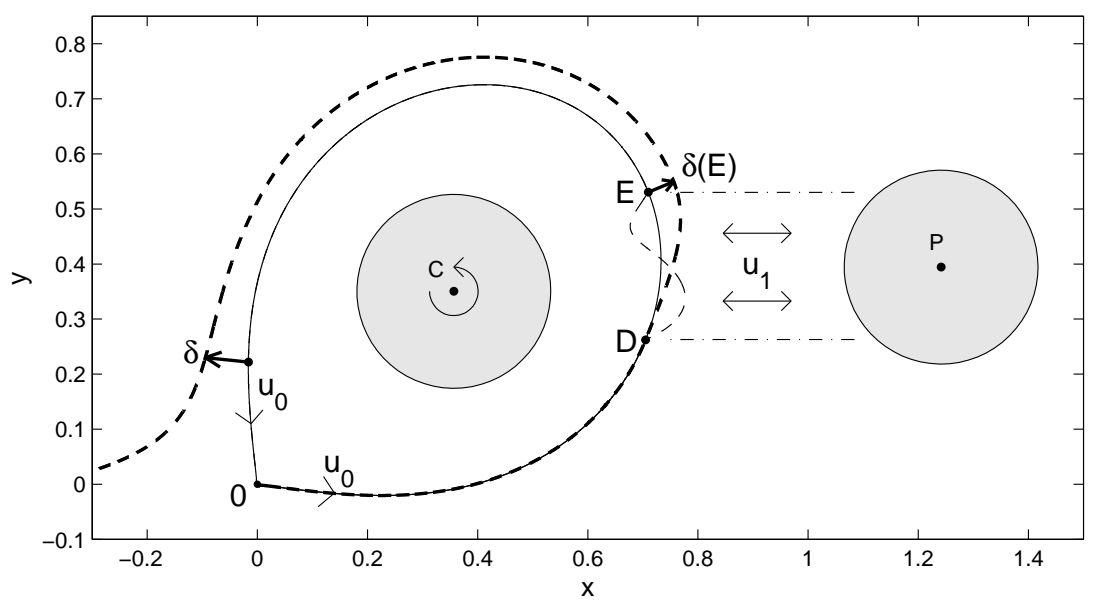

FIG. 7: Schematic of the trajectory of a fluid particle without perturbation (solid line) and with perturbation (dashed lines). The thick (resp. thin) dashed line corresponds to the case where the time for the particle to go from $D$ to $E$ is equal to half an oscillating period (resp. a full period). The distance $\delta$ between the unstable and stable manifold increases close to $O$ due to the divergence of the streamlines.

the flow around $O$ can be Taylor expanded as

$$
\left(\begin{array}{l}
u_{0} \\
v_{0}
\end{array}\right)=\left(\begin{array}{ll}
\gamma & -\Omega \\
\Omega & -\gamma
\end{array}\right)\left(\begin{array}{l}
x \\
y
\end{array}\right)
$$

where the symmetric part $\gamma=4 / \pi$ of the velocity gradient is due to the point vortices and the anti-symmetric part $\Omega=1 / \pi$ reflects the weak background vorticity. The presence of $\Omega$ makes the eigenvectors non-orthogonal (see Fig. 8b) with opposite eigen values $\pm \lambda=$ $\pm \sqrt{\gamma^{2}-\Omega^{2}}$. The flow near $O$ is a 'tilted' stagnation point. The value of the strain $\lambda$ has been measured experimentally by PIV and was found to be very close to the theoretical value (within 1\%). The coordinates $\left(x^{\prime}, y^{\prime}\right)$ of a fluid particle thus evolve exponentially in time as

$$
x^{\prime} \sim e^{\lambda t} \quad \text { and } y^{\prime} \sim e^{-\lambda t} \quad \text { with } \quad \lambda=\sqrt{\gamma^{2}-\Omega^{2}}=\sqrt{15} /(4 \pi) .
$$

As a consequence, the product $x^{\prime} y^{\prime}$ is constant which explains why the maxima and minima are located on a tilted hyperbola. The amplitude of the hyperbola $x^{\prime} y^{\prime}=\chi$ has been 


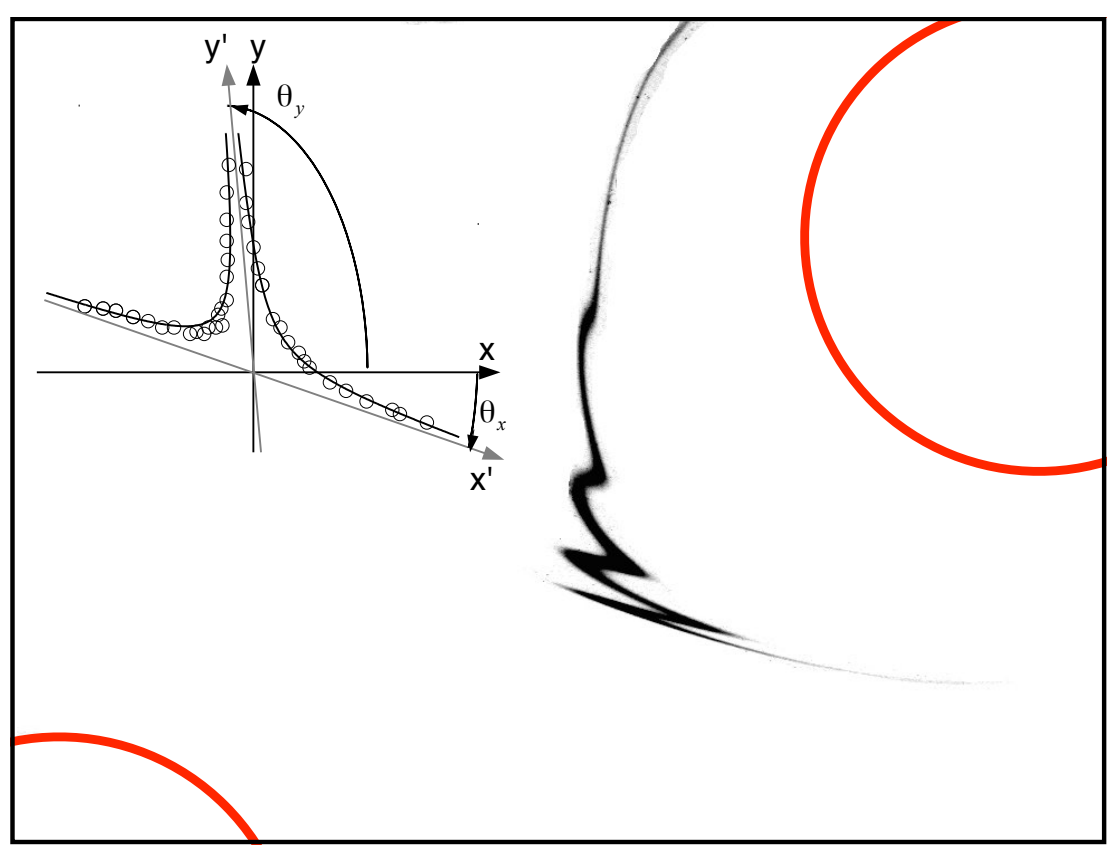

FIG. 8: Dye visualization of the unstable manifold and (insert) position of the maximum and minimum of the dye undulation at different times. The solid line corresponds to the fit with a hyperbola in the new variables $\left(x^{\prime} y^{\prime}=\chi\right) . \omega_{0}=0.5 \mathrm{rad} / \mathrm{s}, \omega_{1}=0.84 \mathrm{rad} / \mathrm{s}$ and $A=0.024$.

measured for each experiment by a least-square fit of the data (with $\chi, \theta_{x}, \theta_{y}$, and the coordinates of the hyperbolic point as fitting parameters). It is plotted in Fig. 9(a) as a function of the amplitude $\epsilon$ of the perturbing cylinder oscillation. It is proportional to the amplitude of the oscillations, which indicates that the experiments are in the linear regime where the Melnikov theory applies.

The Melnikov function $M$ is defined as the distance between the stable and the unstable manifolds multiplied by the velocity of the unperturbed flow. In the schematic view of Fig. 7 where the perturbation is localized between points $D$ and $E$, it corresponds to the product $\delta u_{0}$. This product is exactly equal to the product $\chi \lambda$ we measured previously $\chi=x^{\prime} y^{\prime}$ since $\delta=x^{\prime}$ and $u_{0}=\lambda y^{\prime}$. This is why the product $\chi \lambda$ of the maxima and minima of the undulations should be predicted by the maximum $M_{\max }$ of the Melnikov function.

The main result concerning the Melnikov function is that it can be calculated analytically along the unperturbed trajectory $\mathbf{r}_{0}(t)$ solution of the mean flow $\mathbf{u}_{0}$. Indeed, if the flow is incompressible (i.e. Hamiltonian in dynamical systems), it is given by

$$
M(\tau)=\int_{t=-\infty}^{t=+\infty} \mathbf{u}_{0}\left[\mathbf{r}_{0}(t)\right] \times \mathbf{u}_{1}\left[\mathbf{r}_{0}(t), t+\tau\right] d t
$$

as a function of the phase $\tau$. This formula is the precise formulation of the mechanism explained earlier in Fig. 7 for a localized perturbation where the distance $\delta$ is simply the integral of the normal velocity between points $D$ and $E$. The Melnikov function is of course proportional to $\epsilon$ because $u_{1}$ is proportional to $\epsilon$. Moreover, it is oscillating sinusoidally as a function of the phase $\tau$. The Melnikov function has been calculated numerically by integrating the formula (7) along the trajectory $\mathbf{r}_{0}(t)$. In our case, $\mathbf{r}_{0}(t)$ was also found numerically because it was not possible to find an analytical solution due to the background vorticity term. The maximum $M_{\max }$ for $\tau$ varying over one period is then plotted (thick solid line) as a function of $\omega$ in Fig. 9(b). The Melnikov function increases and presents a peak at $\omega \approx 2$. 


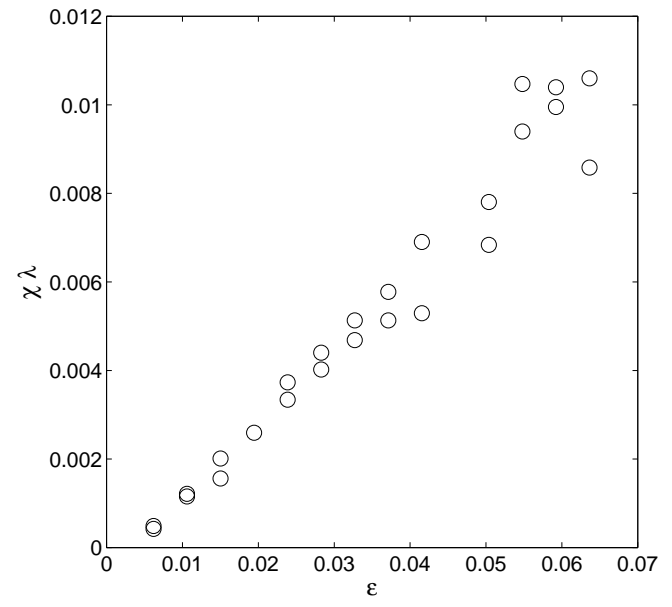

(a)

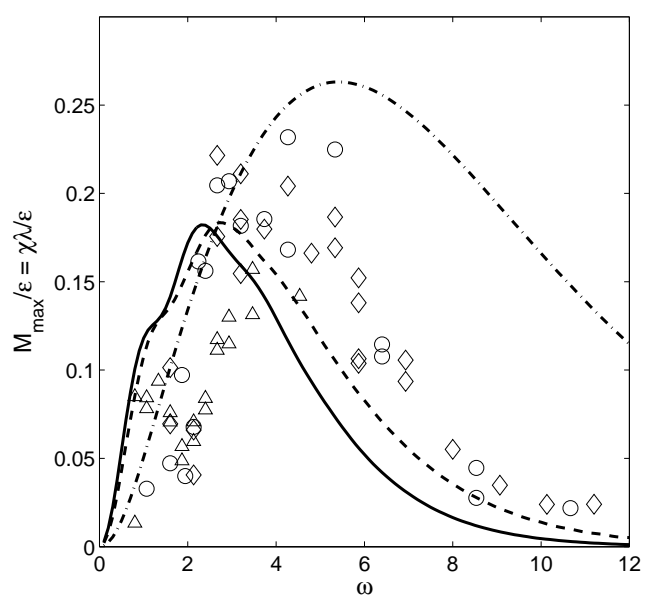

(b)

FIG. 9: Melnikov function as a function of (a) the amplitude and (b) the frequency $\omega$ of the oscillating cylinder. Symbols correspond to experiments at $\omega_{0}=0.5 \mathrm{rad} / \mathrm{s}(\circ), \omega_{0}=1$ $\mathrm{rad} / \mathrm{s}(\square), \omega_{0}=2 \mathrm{rad} / \mathrm{s}(\triangle)$. Lines correspond to the theory without (solid line) and with

(dashed line) the deflection $u_{\text {corr }}$ of the mean flow by the presence of the oscillating cylinder. The dash-dotted line corresponds to the theory given by Eq.(21) for a flow with no background vorticity ${ }^{18}$.

This 'resonance' can be understood in simple terms (see Fig. 7) since $\delta(E)$ is maximum if the time for a particle to go from $D$ to $E$ is equal to half an oscillating period (case of the thick dashed line). In the case where the time to go from $D$ to $E$ is equal to one period (thin dashed line), the distance $\delta(E)$ is always equal to 0 . This is why there is an oscillating frequency $\omega$ where the unstable manifold is most sensitive to the perturbation. Indeed, assuming that the time to travel from $D$ to $E$ is roughly equal to $2 r_{1} / u_{0} \approx 1$, the maximum should be obtained around $\omega \approx \pi$.

This theoretical prediction is compared to the experimental value $\chi \lambda / \epsilon$ measured from the maxima and minima of the tracer dye pattern undulations (Fig. 9). There is a good qualitative agreement since both curves present a maximum. There is a good quantitative agreement on the amplitude of the maximum, which is close to 0.2 in both experiments and theory. However, the maximum is found theoretically for a frequency $\omega \approx 2$ smaller than the experimental observation $\omega \approx 4$ (both values being close to $\pi$, see above). This discrepancy is probably due to the inadequate model of the mean flow. Indeed, the simple addition of the correction $u_{\text {corr }}$ to the mean flow due to the presence of the oscillating cylinder leads to the dashed line which is in better agreement with the experiment. Moreover, it was shown that the background vorticity was damped by the bottom. The extreme case (with zero background vorticity) corresponds to a flow containing only two point vortices ${ }^{18}$. Redoing the calculation for such a flow leads to a theoretical prediction given as a simple integral (see appendix). This prediction is plotted as a dash-dotted line (for $y_{p}=0$ ) in Fig. 9(b). For such a mean flow, the maximum is obtained at $\omega \approx 6$. It is thus possible to tune the background vorticity in order to obtain any agreement between the theory and the experiment. However, such a fit is of no interest for this study, which only aimed at checking the validity of the Melnikov function in a real flow.

Finally, it is interesting to see how the Melnikov function depends on the distance from the cylinder to the hyperbolic point. This was done theoretically by changing the position $x_{P}$ of the perturbing cylinder from 1.15 to 10, as shown on Fig. 10(a). It is clear that the amplitude depends strongly on the distance of the perturbing cylinder, decreasing by a factor 40 when the distance $x_{P}$ increases from 1.15 to 5 . This is natural since the perturbing 


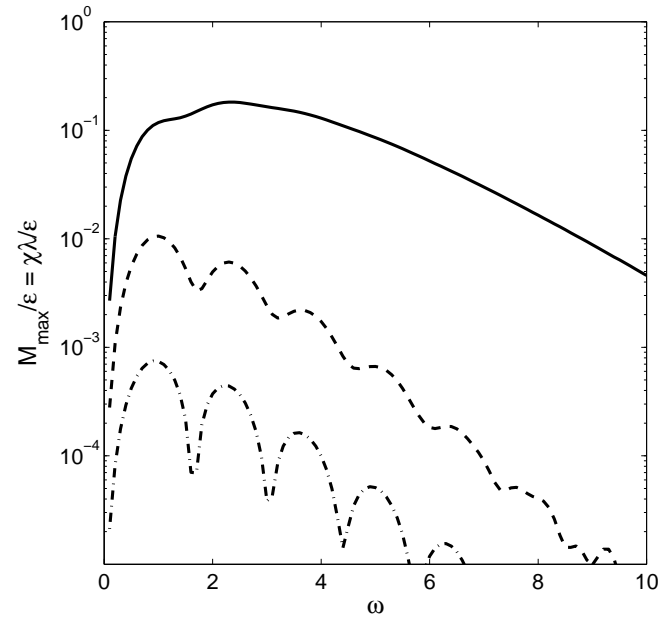

(a)

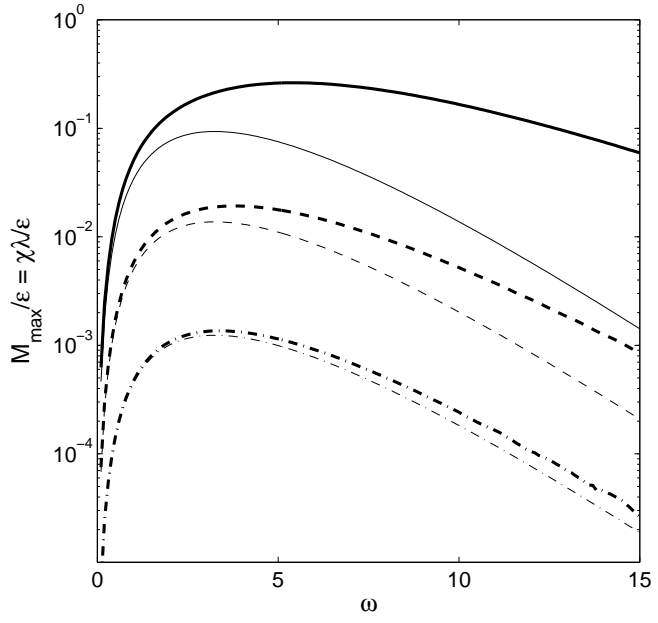

(b)

FIG. 10: Theoretical Melnikov function as a function of the perturbing frequency $\omega$ for various positions of the perturbing cylinder. The solid lines correspond to

$\left(x_{P}, y_{P}\right)=(1.15, .07)$ as in the experiment. Dashed lines correspond to $\left(x_{P}, y_{P}\right)=(3, .07)$ and dash-dotted lines correspond to $\left(x_{P}, y_{P}\right)=(10, .07)$. The theory is calculated in the presence (a) and in the absence of background vorticity. In (b), thick lines correspond to the full theory (21) and thin lines correspond to the approximation of a cylinder located far from the hyperbolic point (8).

velocity field (4) scales as $\left|\mathbf{r}-\mathbf{r}_{P}\right|^{-2}$ such that the Melnikov function should scale as $x_{P}^{-2}$ for large $x_{P}$. Moreover, when the perturbing cylinder is far enough, there are multiple resonant peaks. This can be qualitatively understood by the fact that the fluid particle can spend an odd number of half periods in the interaction zone for the displacement to be maximum. However, it is surprising to see that these multiple peaks disappear in the absence of background vorticity, as shown on Fig. 10(b). This is probably due to the fact that the streamline is at right angle in the case without background vorticity, whereas it has an angle slightly larger than 90 degrees in the presence of background vorticity. When the oscillating cylinder is far from the hyperbolic point, this integral of the Melnikov function can be even calculated analytically, leading to a simple expression for the maximum of the Melnikov function:

$$
M_{\max }=\frac{\epsilon \pi^{2} r_{1}^{2} \omega^{2}}{8 x_{p}^{2} \sqrt{2 \cosh \left(\pi^{2} \omega / 8\right)}} .
$$

This approximate solution is plotted as thin lines in Fig. 10(b)and is close to the full solution when $x_{p}$ is larger than 3 . This simple formula clearly shows that the Melnikov function scales as $x_{P}^{-2}$ at large $x_{P}$.

\section{CONCLUSIONS}

We have studied experimentally the transport properties of a 2D flow with a large mean velocity perturbed periodically in time. The flow was realized using a stratified and viscous fluid in order to ensure perfect 2D motion. We focused on the case of a mean flow containing a homoclinic point perturbed periodically by an oscillating flow. Such a flow is well known to create an undulation of the unstable manifold leading to a large transport of scalar from inside to outside the homoclinic streamline starting from the hyperbolic point. The undulations of the unstable manifold have been measured quantitatively and compared to 
the prediction from the Melnikov theory. The amplitude of the undulations are maximum for a given frequency, which can be estimated by assuming that the mean flow advects a fluid particle over the perturbed area in half a period. There is a good quantitative agreement between the theory and the experiment, given that there is no fitting parameter in the model. However, the resonant frequency is slightly underestimated by the theory probably due to small corrections to the mean flow that have not been taken into account.

This study interestingly underlines the effect of a small perturbation on the transport properties of a mean flow. Moreover, it validates the Melnikov theory which has been widely used in the literature but which has never been tested experimentally. The first reason is probably that it is difficult to create a 2D flow. This technical problem has been solved here by a subtle combination of salt and $\mathrm{UCON}^{\mathrm{TM}}$ oil in order to create a viscous stratified fluid. The second reason is also that it is difficult to create a flow with a homoclinic point (or two heteroclinic points ${ }^{11}$ ). This flow topology has been realized here by two co-rotating cylinders, which lead to a flow predicted by a simple theory with no adjustable parameter. This is a major advantage since it allows to test the Melnikov theory accurately.

The initial goal of this study was to quantify the transport of dye from inside to outside the streamline starting from the homoclinic point. Indeed, the Melnikov theory can be used to predict the surface of the lobes of the unstable manifold and thus to evaluate the amount of dye which exits the streamline. However, experiments have shown that the position of injection of the dye was critical for the late evolution of the dye. If the dye was located too far from the central streamline of the contracting direction, it would become homogenized by diffusion in a band that would never reach the hyperbolic point. If the dye was located too close from the streamline, it would exit the streamline very rapidly in the stretching direction, before being homogenized on the inner part of the streamline, thus preventing the measurement of the amount of dye exiting the streamline. However, these results indicate that the transport of dye from inside to outside this streamline is extremely rapid close to the streamline. The global transport at late stages is thus limited by the slow advectiondiffusion far from the streamline (i.e. close to the vortex centers), which is strongly damped by the presence of KAM torii. It thus seems that the study of advection-diffusion in a weakly perturbed vortex is of primary importance for the understanding of $2 \mathrm{D}$ mixing, an extension of this study that we leave for future research.

\section{ACKNOWLEDGEMENTS}

The authors would like to than the french "Agence Nationale de la Recherche" for the funding of the ANR-DFG TurbMix.

\section{APPENDIX}

The goal is to calculate the Melnikov function for the flow of two point vortices (without background vorticity) whose streamfunction is given by

$$
\Psi_{0}=\frac{1}{4 \pi}\left(\log \left|\mathbf{r}-\mathbf{r}_{\mathbf{C}}\right|+\log \left|\mathbf{r}-\mathbf{r}_{\mathbf{C}^{\prime}}\right|\right) .
$$

The calculation is similar to the calculation done by Raynal and Gence $(1995)^{18}$ but for a different perturbation. We also perform the calculation with different axes (rotated by 45 degrees with respect to their axes). The details of the calculation are different but the method is very similar and we will only give the main steps here.

In the absence of perturbation, the homoclinic trajectory (starting at $O$ ) is defined by the fact that the streamfunction is constant and equal to $\log (1 / 16) /(2 \pi)$. Introducing this value into (9) gives, on the homoclinic trajectory, the following relation

$$
\left|\mathbf{r}-\mathbf{r}_{\mathbf{C}}\right|^{2}\left|\mathbf{r}-\mathbf{r}_{\mathbf{C}^{\prime}}\right|^{2}=\frac{1}{16}
$$


which can be simplified into

$$
\left(x^{2}+y^{2}\right)^{2}=x y
$$

The mean velocity on this trajectory can be simplified using (10) to replace the fractions by polynomials whose degree can be further reduced by using (11). This leads to simple differential equations for the homoclinic trajectory:

$$
\begin{aligned}
& \dot{x}=\frac{4}{\pi}\left(x-4 y\left(x^{2}+y^{2}\right)\right) \\
& \dot{y}=-\frac{4}{\pi}\left(y-4 x\left(x^{2}+y^{2}\right)\right)
\end{aligned}
$$

Introducing the new variable $p=x^{2}+y^{2}$ allows to get a $1 \mathrm{D}$ differential equation

$$
\begin{aligned}
\frac{d p}{d t} & =2 x \frac{d x}{d t}+2 y \frac{d y}{d t} \\
& =\frac{8}{\pi}\left(x^{2}-y^{2}\right) \\
& = \pm \frac{8}{\pi} \sqrt{p^{2}-4 p^{4}}
\end{aligned}
$$

Here, we have used (11) to calculate $\left(x^{2}-y^{2}\right)^{2}=\left(x^{2}+y^{2}\right)^{2}-4(x y)^{2}$ as a function of $p$. Introducing the new variable $q=1 /(2 p)$ leads to a simple differential equation whose solution is $q=\cosh ( \pm 8 t / \pi)$ from which $p$ can be evaluated as:

$$
\begin{aligned}
p & =x^{2}+y^{2} \\
& =\frac{1}{2 \cosh ( \pm 8 t / \pi)}
\end{aligned}
$$

Using (11), it is easy to evaluate $(x+y)^{2}$ and $(x-y)^{2}$ which can be simplified using trigonometric functions such that

$$
\begin{aligned}
& x+y=\frac{\cosh ( \pm 4 t / \pi)}{2 \cosh ( \pm 8 t / \pi)} \\
& x-y=\frac{\sinh ( \pm 4 t / \pi)}{2 \cosh ( \pm 8 t / \pi)}
\end{aligned}
$$

from which $x$ and $y$ are derived as

$$
\begin{aligned}
& x=\frac{\exp (-4 t / \pi)}{2 \cosh (8 t / \pi)} \\
& y=\frac{\exp (4 t / \pi)}{2 \cosh (8 t / \pi)} .
\end{aligned}
$$

Here, the signs have been chosen to follow the trajectory around $C$ in the right direction. The velocity of the perturbation is given by (4) which can be rewritten as

$$
\mathbf{u}_{1}(x, y, t+\tau)=\frac{\epsilon \omega r_{1}^{2} \cos (\omega(t+\tau))}{\left|\mathbf{r}-\mathbf{r}_{p}\right|^{4}}\left(\begin{array}{l}
\left(x-x_{p}\right)^{2}-\left(y-y_{p}\right)^{2} \\
2\left(x-x_{p}\right)\left(y-y_{p}\right)
\end{array}\right) .
$$

The Melnikov function defined by (7) since $\mathbf{u}_{0}$ given by (12) can thus be evaluated as a simple integral with polynomials of degree 5 in $x$ and $y$ at the numerator and of degree 4 at the denominator. Using the relation (11), the degree of these polynomials can be reduced to 3. Introducing the temporal dependence of $x$ and $y$ from (16) leads to a rational fraction in $e^{4 t / \pi}$ which imposes the change of variable

$$
s=e^{4 t / \pi} \quad \text { and thus } \quad d s=\frac{4 s}{\pi} d t .
$$


Using the fact that

$$
\begin{aligned}
\cos (\omega(t+\tau)) & =\operatorname{Re}\left(e^{i \omega t}\right) \cos (\omega \tau)-\operatorname{Im}\left(e^{i \omega t}\right) \sin (\omega \tau) \\
& =\operatorname{Re}\left(s^{i \omega \pi / 4}\right) \cos (\omega \tau)-\operatorname{Im}\left(s^{i \omega \pi / 4}\right) \sin (\omega \tau)
\end{aligned}
$$

where $\operatorname{Re}(\cdot)$ and $\operatorname{Im}(\cdot)$ denote real and imaginary parts, the Melnikov function is thus written

$$
M(\tau)=\operatorname{Re}(I) \cos (\omega \tau)-\operatorname{Im}(I) \sin (\omega \tau)
$$

with

$$
\begin{aligned}
& \frac{I}{\epsilon \omega r_{1}^{2}}= \\
& \int_{0}^{\infty} \frac{s^{2}\left(4 s x_{p}-s^{2}+\left(s^{4}-3\right) x_{p}^{2}\right)+2 y_{p}\left(s^{5}-s+\left(1-3 s^{4}\right) x_{p}\right)-s^{2} y_{p}^{2}\left(s^{4}-3\right)}{\left(s^{2}-2 s x_{p}+x_{p}^{2}-2 s^{3} y_{p}+y_{p}^{2}+s^{4}\left(x_{p}^{2}+y_{p}^{2}\right)\right)^{2}} s^{i \omega \pi / 4} d s .
\end{aligned}
$$

The maximum of the Melnikov function is obtained for $\tan (\omega \tau)=\operatorname{Im}(I) / \operatorname{Re}(I)$ and is equal to $M_{\max }=|I|$. The integral can be calculated analytically when the oscillating cylinder is far from the hyperbolic point, i.e. by assuming that $x_{p}$ is much larger than $x, y$ and $y_{p}$, leading to

$$
M_{\max }=\frac{\epsilon \pi^{2} r_{1}^{2} \omega^{2}}{8 x_{p}^{2} \sqrt{2 \cosh \left(\pi^{2} \omega / 8\right)}}
$$

${ }^{1}$ A. D. Stroock, S. K. W. Dertinger, A. Ajdari, I. Mezic, H. A. Stone, and G. M. Whitesides, "Chaotic mixer for microchannels," Science 295, 647-651 (2002).

${ }^{2}$ E. Villermaux, A. D. Stroock, and H. A. Stone, "Bridging kinematics and concentration content in a chaotic micromixer," Phys. Rev. E 77 (2008), 10.1103/PhysRevE.77.015301.

${ }^{3}$ S. Wiggins, "The dynamical systems approach to Lagrangian transport in oceanic flows," Ann. Rev. Fluid Mech. 37, 295-328 (2005).

${ }^{4}$ B. Joseph and B. Legras, "Relation between kinematic boundaries, stirring, and barriers for the antarctic polar vortex," J. Phys. Oceanography 59, 295-328 (2002).

${ }^{5}$ P. Haynes and E. Shuckburgh, "Effective diffusivity as a diagnostic of atmospheric transport: 1. stratosphere," J. Phys. Oceanography 105 (2000).

${ }^{6}$ T.-Y. Koh and R. A. Plumb, "Lobe dynamics applied to barotropic Rossby-wave breaking," Phys. Fluids 12, 1518-1528 (2000).

${ }^{7}$ A. Provenzale, "Transport by coherent barotropic vortices," Ann. Rev. Fluid Mech. 31, 55-93 (1999).

${ }^{8}$ T. Shepherd and R. A. Plumb, "The antarctic vortex splitting event," J. Atmos. Sci., Special Issue 62 (2005).

${ }^{9}$ F. Lekien and S. D. Ross, "The computation of finite-time lyapunov exponents on unstructured meshes and for non-Euclidean manifolds," Chaos 20, 017505 (2010).

${ }^{10}$ J. M. Ottino, The Kinematics of Mixing: Stretching, Chaos, and Transport (Cambridge University Press., 1989).

${ }^{11}$ V. Rom-Kedar, A. Leonard, and S. Wiggins, "An analytical study of transport, mixing and chaos in an unsteady vortical flow," J. Fluid Mech 214, 347-394 (1990).

${ }^{12}$ D. Beigie, A. Leonard, and S. Wiggins, "A global study of enhanced stretching and diffusion in cahotic tangles," Phys. Fluids A 3, 1039-1050 (1991).

${ }^{13}$ J. Guckenheimer and P. Holmes, Non-Linear Oscillations, Dynamical Systems and Bifurcations of Vector Fields (Springer, 1983).

${ }^{14} \mathrm{~S}$. Wiggins, Introduction to Applied Nonlinear Dynamical Systems and Chaos (Springer, 2003).

${ }^{15}$ T. Solomon, A. T. Lee, and M. Fogleman, "Resonant flights and transient superdiffusion in a timeperiodic, two-dimensional flow," Physica D: Nonlinear Phenomena 157, 40 - 53 (2001).

${ }^{16}$ R. Camassa and S. Wiggins, "Chaotic advection in a Rayleigh-Benard flow," Phys. Rev. A 43, 774-798 (1991).

${ }^{17}$ R. M. Samelson, "Fluid exchange across a meandering jet," J. Phys. Oceanography 22, 431-440 (1992).

${ }^{18}$ F. Raynal and J. N. Gence, "Efficient stirring in planar, time-periodic laminar flows," Chem. Eng. Science 50, 631-640 (1995).

${ }^{19}$ K. Ngan and T. G. Shepherd, "Chaotic mixing and transport in rossby-wave critical layers," J. Fluid Mech. 334, 315-351 (1997).

${ }^{20}$ D. del Castillo-Negrete, "Asymmetric transport and non-gaussian statistics of passive scalars in vortices in shear," Phys. Fluids 10, 576-590 (1998).

${ }^{21}$ V. Rom-Kedar and A. C. Poje, "Universal properties of chaotic transport in the presence of diffusion," Phys. Fluids 11, 2044 (1999). 
${ }^{22}$ M. Brons, W. Z. Shen, J. N. Sorensen, and W. J. Zhu, "The influence of imperfections on the flow structure of steady vortex breakdown bubbles," J. Fluid Mech. 578, 453-466 (2007).

${ }^{23}$ M. Brons, M. Thompson, and K. Hourigan, "Dye visualization near a three-dimensional stagnation point: application to the vortex breakdown bubble," J. Fluid Mech. 622, 177-194 (2009).

${ }^{24}$ J. M. Lopez and A. D. Perry, "Axisymmetric vortex breakdown. part 3 onset of periodic flow and chaotic advection," J. Fluid Mech 234, 449-471 (1992).

${ }^{25}$ P. Meunier and K. Hourigan, "Mixing in a vortex breakdown flow," J. Fluid Mech. 731, 195-222 (2013).

${ }^{26}$ W.-L. Chien, H. Rising, and J. M. Ottino, "Laminar mixing and chaotic mixing in several cavity flows," J. Fluid Mech. 170, 355-377 (1986).

${ }^{27}$ J. Chaiken, C. R., M. Tabor, and Q. M. Tan, "Experimental study of lagrangian turbulence in stokes flow," Proc. R. Soc. Lond. A 408, 165-174 (1986).

${ }^{28}$ J. M. Ottino, C. W. Leong, R. H., and P. D. Swanson, "Morphological structures produced by mixing in chaotic flows," Nature 333, 419-425 (1988).

${ }^{29}$ P. D. Swanson and J. M. Ottino, "A comparative computational and experimental study of chaotic mixing of viscous fluids," J. Fluid Mech. 213, 227-249 (1990).

${ }^{30}$ S. C. Janat, G. Metcalfe, and J. M. Ottino, "Experimental and computational studies of mixing in complex Stokes flows: the vortex mixing flow and multicellular cavity flows," J. Fluid Mech. 269, 199246 (1994).

${ }^{31}$ H. Aref, "Stirring by chaotic advection," J. Fluid Mech. 143, 1-21 (1984)

${ }^{32}$ T. H. Solomon and J. P. Gollub, "Chaotic particle transport in time-dependent Rayleigh-Bénard convection." Phys. Rev. A 38, 6280-6286 (1988).

${ }^{33}$ T. H. Solomon, S. Tomas, and J. L. Warner, "Role of lobes in chaotic mixing of miscible and immiscible impurities," Phys. Rev. Lett. 77, 2682 (1996).

${ }^{34}$ D. Rothstein, E. Henry, and J. P. Gollub, "Persistent patterns in transient chaotic fluid mixing," Nature 401, 770-772 (1999).

${ }^{35}$ B. Williams, D. Marteau, and J. Gollub, "Mixing of a passive scalar in magnetically forced twodimensional turbulence," Phys. Fluids 9, 2061 (1997).

${ }^{36}$ G. A. Voth, T. C. Saint, G. Dobler, and J. P. Gollub, "Mixing rates and symmetry breaking in twodimensional chaotic flow," Phys. Fluids 15, 2560 (2003).

${ }^{37}$ L. Rossi, J. C. Vassilicos, and Y. Hardalupas, "Electromagnetically controlled multi-scale flows," J. Fluid Mech. 558, 207-242 (2006).

${ }^{38}$ A. Figueroa, P. Meunier, S. Cuevas, E. Villermaux, and E. Ramos, "Chaotic advection at large Péclet number: Electromagnetically driven experiments, numerical simulations, and theoretical predictions," Phys. Fluids 26, 013601 (2014).

${ }^{39}$ D. H. Kelley and N. T. Ouellette, "Onset of three-dimensionality in electromagnetically driven thin-layer flows," Phys. Fluids 23, 045103 (2011).

${ }^{40}$ J. Paret, D. Marteau, O. Paireau, and P. Tabeling, "Are flows electromagnetically forced in thin stratified layers two dimensional?" Phys. Fluids 9, 3102 (1997).

${ }^{41}$ T. Solomon and I. Mezic, "Uniform resonant chaotic mixing in fluid flows," Nature 425, 376-380 (2003).

${ }^{42}$ P. Meunier and E. Villermaux, "Van Hove singularities in Probability Density Functions of scalars," C. R. Mecanique 335, 162-167 (2007).

43 UCON 75-H-90,000, Available from DOW Company, Michigan, USA (2015).

${ }^{44} \mathrm{P}$. Meunier and T. Leweke, "Analysis and optimization of the error caused by high velocity gradients in PIV," Exp. Fluids 35, 408-421 (2003).

${ }^{45}$ H. Lamb, Hydrodynamics, 6th edition. (Cambridge University Press., 1932). 